\title{
Psychological Effects of Secondary Prisonization on Older Parents
}

\author{
Luixa Reizabal ${ }^{1}(1) \cdot$ Iñaki Garcia ${ }^{2}(1) \cdot$ Eneko Sansinenea $^{2}(1)$.

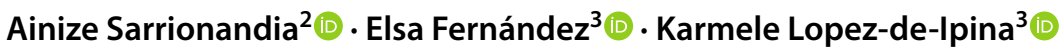

Accepted: 18 November 2021 / Published online: 7 December 2021

(c) The Author(s) 2021

\begin{abstract}
Secondary prisonization refers to the impact of the incarceration of a relative on the members of their family. This study aimed to analyze the psychological effects of secondary prisonization on older parents. Specifically, levels of depression, anxiety, stress, and well-being (emotional, psychological, and social) were analyzed by means of quantitative and automatic speech analysis methods in a sample of over 65 -year-old parents of Basque prisoners incarcerated in remote prisons. The statistical analysis of data and the automatic spontaneous speech analysis showed that secondary prisonization has a negative impact on older parents' levels of depression, anxiety, stress, and well-being. These results lead us to conclude that remote imprisonment of adult children has negative psychological effects on older parents.
\end{abstract}

Keywords Secondary prisonization · Older parents · Mental health · Depression · Anxiety $\cdot$ Stress $\cdot$ Well-being

\section{Introduction}

The present study analyzes the effects of secondary prisonization on older parents of Basque prisoners incarcerated in Spain. Secondary prisonization refers to the impact of a relative's incarceration of on the members of their family (Comfort, 2003). This is an important and contemporary question, even more so if we consider that, according to the World Prison Brief online database (2021) hosted by

Ainize Sarrionandia

ainize.sarrionandia@ehu.eus

1 Department of Basic Psychological Processes and Development, University of the Basque Country, Basque Country, Donostia-San Sebastian, Spain

2 Department of Clinical Psychology, Health Psychology and Research Methodology, University of Basque Country, Basque Country, Donostia-San Sebastian, Spain

3 Department of Systems Engineering and Automation, University of Basque Country, Basque Country, Donostia-San Sebastian, Spain 
the Institute for Crime and Justice Policy Research, Spain has one of the highest incarceration rates in the European Union, with a prison population rate of 122 per 100,000 of national population.

Article 12 of the General Penitentiary Law of 5 October 1979 establishes that the location of prisons shall avoid the social uprooting of prisoners. Therefore, Spanish prisoners incarcerated in Spain must serve their sentences in prisons located close to their homes and those of their families. However, as a result of the specific penitentiary regime applied to Basque prisoners incarcerated for political reasons, these prisoners serve their sentences in prisons located far from their homes and those of their families. This situation restricts the right to respect for private and family life (Article 8 of the European Human Rights Convention) as was stated by the European Court of Human Rights in cases such as the Rodzevillo sentence against Ukraine, of 14 January 2016.

The effects on relatives of incarceration close to the places of residence of prisoners and their families have been studied, but little is known about the effects of remote incarceration on prisoners' relatives. Consequently, the present study attempted to shed light on the psychological effects of secondary prisonization on older parents of Basque prisoners incarcerated in remote prisons.

As mentioned, secondary prisonization refers to the effects that imprisonment of a family member has on their relatives (Comfort, 2003). Loss of rights and resources, as well as the negative psychological and social effects on family members of being a prisoner's relative, are included in secondary prisonization. In relation to psychological effects, five domains have been identified (Observatori del Sistema Penal i els Drets Humans, 2006): psychological disorders, those related to affectivity (loneliness, lack of support), low self-esteem, distorted perception of the world, and defenselessness and hostility towards penitentiary institutions.

One important dimension of secondary prisonization is mental health. Traditionally, mental health has been conceptualized as absence of symptoms of psychological disorders. This conceptualization of mental health is known as the pathogenic approach and aims to identify, avoid, manage, and cure illness (Taylor, 1990).

Although the pathogenic approach has traditionally been the main position related to mental health, mental health is now considered “... a state of wellbeing in which the individual realizes his or her own abilities, can cope with the normal stresses of life, can work productively and fruitfully, and is able to make a contribution to his or her community" (World Health Organization, 2001:1). This definition reflects the salutogenic approach to mental health, which aims to gain knowledge about mental health and the factors that determine it.

An integrative model of mental health has recently been proposed. This model is called The Two Continua Model of Mental Health (Keyes, 2005), which brings together both pathogenic and salutogenic perspectives of mental health. According to this model, mental health and mental illness would not be the opposite ends of a single continuum, but, rather, two related continua: the psychopathological continuum and the positive mental health continuum. The psychopathological continuum relies on the pathogenic perspective, where common psychiatric and clinical disorders and symptoms are identified. The Positive Mental Health continuum (Keyes, 2002) refers 
to a dimension of mental health composed of emotional, psychological, and social human well-being, and it describes the salutogenic view of health (Keyes, 2002).

Emotional well-being refers to the total amount of positive emotions and satisfaction with life an individual can have. Psychological well-being, instead, refers to six aspects of the way a person lives their life: (a) self-acceptance; (b) the affective quality of close relationships; (c) feelings of personal growth; (d) the perception of purpose in life; (e) the mastery of the personal environment; and (f) the capacity or autonomy to defend their own ideas when they do not form part of general beliefs (Keyes, 2002).

The third component of positive mental health is social well-being. This dimension refers to the perceived fit between a person and society. Five variables describe this dimension: (a) social integration is the perception that a person has something in common with the rest of people and that they belongs to a society; (b) social contribution refers to how a person feels they have something to offer to their fellow citizens; (c) social coherence is the perception that society is coherent and knowable or understandable; (d) social growth is the perception that society has a positive trajectory - the overall sense that, despite social conflicts and problems, people have improved society; and (e) social acceptance is the acknowledgment of positive qualities in the average citizen, qualities that include trustworthiness, altruism and industriousness (Keyes, 2002).

Thus, instead of understanding well-being as the same as pleasure (emotional well-being), positive mental health proposes that an overall comprehension of wellbeing requires taking into account the way a person lives their life (psychological well-being) and the way a person perceives society and their own role within it (social well-being).

The psychopathological and the positive mental health continua are independent, that is, they are created by different factors and go in different directions. For instance, a person might have a psychiatric problem, and thus, this person would be situated "high" on the psychopathological dimension of mental health, but would also have good positive mental health, that is, they are hopeful in regard to their problem and are completely committed to their recovery. In another case, a person could be mentally healthy in the sense that they have no mental health diagnosis, but may feel discouraged, finding no meaning to their life, and experience very little joy and happiness.

As these two continua are unrelated, the relationship between them is low (a correlation of -0.53 in a study conducted with a large sample; these continua share only $28.1 \%$ of variance; Keyes, 2005).

Based on this theoretical point of view, the present research aims to analyze the effects of secondary prisonization on levels of psychopathology (depression, anxiety, and stress) and positive mental health (psychological, social, and emotional well-being) in a sample of over 65-year-old parents of prisoners incarcerated at a great distance from the Basque Country.

Various studies have addressed the effects of secondary prisonization on prisoners' relatives incarcerated close to their residences. These studies have mainly been carried out with prisoners' children and partners (Aiello \& McCorkel, 2018; Comfort, 2003; Murray et al., 2012). In contrast, few studies have been conducted in relation to the effects of secondary prisonization on prisoner's parents. One of these studies 
was carried out with a sample of prisoners' mothers, and it was found that having a son/daughter in jail was related to their mothers' psychological suffering (Green et al., 2006).

In all the aforementioned studies, the samples were of relatives of prisoners incarcerated in prisons close to their and their families' places of residence. The only study where the effects of remote incarceration on family members have been examined was conducted by Intxaurbe et al. (2017). In this study, a sample of 14 prisoners' relatives (parents, partners, children, brothers, and sisters) were interviewed, and it was found that remote incarceration entails the suffering of prisoner's relatives, mainly when they are older adults.

\section{Objective}

In order to fill the gap in the literature on the effects of secondary prisonization on relatives of prisoners incarcerated in remote prisons, the present study had a primary objective: to analyze the psychological effects of secondary prisonization on older parents of Basque prisoners. Specifically, the effects on levels of depression, anxiety, stress, and well-being (emotional, psychological, and social) were analyzed. With this aim in mind, we compared the two continua of mental health (psychopathology and positive mental health) in the case of older parents of Basque prisoner's and older parents from the general Basque population.

Thus, two hypotheses were proposed. Firstly, it was hypothesized that, compared with older parents from the general population, Basque prisoners' older parents would show higher levels of psychopathology. Specifically, it was expected that older parents of Basque prisoners would show higher levels of depression, anxiety, and stress. Secondly, it was hypothesized that, compared with older parents from the general population, those of Basque prisoners would show lower levels of positive mental health. Specifically, older parents of Basque prisoners were expected to show lower levels of emotional, psychological, and social well-being.

\section{Methods}

\section{Participants}

To achieve the main objective of the study, two groups were studied: older parents with a son/daughter in a remote prison and older parents without sons/daughters in prison. Non-random sampling was used to obtain these groups. The inclusion criteria for the first group were the following: being older than 65; having a daughter/son incarcerated for political reasons; having a daughter/son incarcerated in a remote prison; living in the Basque Country; giving informed consent. The exclusion criteria for this sample were as follows: being younger than 65 ; having more than one daughter/son in prison; having a daughter/son incarcerated for reasons unrelated to politics; having a daughter/son in a prison near to their place of residence; not giving informed consent. 
In relation to the second sample, the inclusion criteria were the following: being older than 65; not having a daughter/son in prison; living in the Basque Country; and giving informed consent. The exclusion criteria for this group were as follows: being younger than 65; having a daughter/son in prison; not giving informed consent.

To obtain the sample of prisoners' parents, researchers contacted Etxerat, an association of family members and friends of Basque prisoners and refugees. Etxerat provided the researchers with the contact details of older parents with a daughter or son incarcerated in remote prisons. The daughters and sons of these parents had spent an average of 13 years $(S D=4.65)$ in prisons which are approximately $674 \mathrm{~km}(S D=242.44)$ from their homes.

Regarding the representativeness of this group, at the time of the study, 254 Basque prisoners were incarcerated in remote jails. Most of these prisoners had only one parent alive and more than $90 \%$ of prisoners' parents were older than 65 (Etxerat, 2018). Thus, around 228 parents were more than 65 years old. Thus, the sample of the present study represented $20 \%$ of the total sample.

In relation to the sample of parents aged over 65 with no daughters/sons in prison, researchers went to retirement homes and randomly asked residents to participate in the study.

The present study thus consisted of 90 individuals: 59 females $(65.6 \%)$ and 31 males $(34.4 \%)$. The mean age of participants was $71.9(S D=6.45)$. Specifically, the mean age of the prisoners' parents group was $71.4(S D=5.7)$ and the mean age of the controls was $72.4(S D=7.1)$. Regarding marital status, most participants were married (64.4\%), almost a third of the sample were widowed $(27.8 \%)$ and a few were divorced $(4.4 \%)$ or unmarried (3.3\%). As for educational level, most of them had completed primary or secondary education $(45.6 \%$ and $28.9 \%$ respectively), while a minority who had higher education qualifications (17.8\%) or no studies $(7.8 \%)$.

No significant differences were found between the two groups regarding age $[t(88)=-0.73 ; p=0.46]$, gender $\left.\left[\chi^{2}(1, n=90)=0.04 ; p=0.82\right)\right]$ and education level $\left.\left[\chi^{2}(1, n=90)=0.57 ; p=0.90\right)\right]$.

Ethical Issues and Procedure.

To carry out the present study, approval from the Human Research Ethics Committee of the researchers' University was obtained (M10/2018/167).

Once the approval was obtained, a group of psychologists was recruited and trained to interview participants individually. Before the interview, participants were informed about the research and gave their written consent to participate. The interviews were carried out in a calm atmosphere and in a positive and open dialog. They lasted approximately $30 \mathrm{~min}$.

The interviews had two parts. In the first one, participants were asked to answer a questionnaire in order to operationalize the variables that will be explained in the next section. In the second part, each participant was asked to talk about something positive that had happened to him/her and this was recorded on audio. In this way, 5 min of spontaneous positive speech was obtained. 


\section{Instruments}

Besides sociodemographic variables and the distance from the prison to the parents' home, psychopathology (depression, stress, and anxiety) and positive mental health (psychological, social, and emotional well-being) were assessed. To this end, two mental health measurement methods were applied: the conventional questionnaire method and the automatic analysis of spontaneous speech. In this way, researchers had the chance to test differences in the way both measurements tackle the problem of psychopathology and positive mental health assessment.

The tools used to assess sociodemographic variables, distance from the prison to parents' home, psychopathology (depression, stress, and anxiety), and positive mental health (psychological, social, and emotional well-being) were the following:

Sociodemographic variables: an ad hoc questionnaire was administered to gather data on age, gender, marital status, and education level.

Distance to home: parents were asked where their children were incarcerated in order to measure the distance between the prison and the parents' home.

Depression. The Beck Depression Inventory-Second Edition (BDI-II) was the tool chosen to evaluate depression (Beck et al., 1996; Spanish version by Sanz \& Vázquez, 2011). The BDI-II is composed of 21 items that measure various depressive symptoms, such as sadness, crying, guilt, and suicide ideation. The symptoms measured with the BDI-II coincide with the criteria used by the DSM-5 and ICD-11 (American Psychiatric Association, 2013; World Health Organization, 2018). Each item of the questionnaire has four response options (0-3), depending on the severity of the symptoms. However, items 16 and 18 present seven options. For the interpretation of results, scores from 0 to 13 correspond to no depression; 14-19 means weak depression; 20-28 represents moderate depression; and 29-63 indicates severe depression. Regarding psychometric properties, the Spanish version of the BDI-II presents adequate validity and reliability (Sanz \& Vázquez, 2011), presenting Cronbach's alphas of above 0.85. Other studies have also validated the BDI-II in Spanish samples, demonstrating good psychometric properties of the Spanish version. In fact, the instrument has been validated in university samples (Sanz et al., 2003a, b) as well as in the general population (Sanz et al., 2003a, b) and in patients with psychological disorders (Sanz et al., 2005) showing adequate reliability and validity.

Anxiety. The Goldberg Anxiety and Depression Scale (GADS) was used to evaluate anxiety (Goldberg et al., 1988; Spanish version of Duch et al., 1999). The GADS evaluates depression and anxiety and provides information about their severity. The instrument asks about the presence and severity of symptoms in the last 2 weeks. The two subscales that comprise the instrument (depression and anxiety) have nine items where the participant is asked to say whether or not they have experienced the symptom. The anxiety subscale was used in the present study. The cut-off point is four and the higher the score, the greater is the anxiety. The instrument presents adequate psychometric properties. In particular, and regarding reliability, the GADS showed an $81 \%$ internal consistency by Cronbach's alpha coefficient for the full scale, 0.70 for the depression scale and 0.74 for the anxiety scale (Mackinnon et al., 1994). Likewise, the generalized anxiety disorder instrument has shown proper 
psychometric properties in its Spanish adaptation with a Cronbach's alpha of 0.936 (García-Campayo et al., 2010).

Stress. The Perceived Stress Scale (PSS) was used to evaluate stress (Cohen et al., 1983; Spanish version of Remor, 2006). This self-report instrument measures perceived stress over the last month. The PSS has 14 items scored on a range from 0 (never) to 4 (almost always). The higher the score, the greater is the perceived stress. The PSS-14 presents appropriate psychometric properties. Regarding internal reliability, Cronbach's alpha is 0.81 and the test-retest coefficient $r=0.73$. Likewise, in a study conducted by Pedrero-Pérez et al. (2015), the PSS-14 showed good psychometric properties in the Spanish validation of the instrument, with a Cronbach's alpha of 0.86 in the non-clinical sample and 0.89 in the clinical sample.

Positive Mental Health. Positive mental health was measured by means of the Mental Health Continuum Short Form (MHC-SF) (Keyes, 2005; Spanish version of Peña et al., 2017). The MHC-SF measures positive mental health with 14 items scored on a range from 1 (never) to 5 (always). It measures three dimensions: emotional mental health (three items measuring the frequency of happiness/wellbeing of the last month), social mental health (five items measuring variables such as social contribution or social acceptance), and psychological mental health (five items measuring positive relations, personal growth). A total score is obtained summing all items. The higher the score, the better is the respondent's mental health. The MHC-SF presents adequate psychometric properties. In particular, the instrument presents good internal consistency (Cronbach alpha $>0.80$; in the Spanish version: 0.91). Regarding construct validity, results are promising. In fact, negative correlations have been found between depression and anxiety (Keyes, 2002, 2005). In the same line, applied in a Spanish context, the instrument has shown good psychometric properties for both the global scale and the different dimensions, with a Cronbach's alpha above 0.80 in all the dimensions (Olivos et al., 2014).

Automatic analysis of spontaneous speech. Language is the most natural way of communication, and its deterioration is often a symptom of illness. Therefore, the analysis of oral communication has emerged as a useful tool to detect cognitive and physiological variations. It is easy to use, effective, cheap, and non-invasive. The speech message, the way of speaking or the silences a person makes, undoubtedly indicates the individual's cognitive and physiological variations. Moreover, oral communication can be a powerful biomarker of mental health and emotional state. Indeed, oral communication is one of the first skills lost in certain neurodegenerative diseases (Alzheimer's disease, Parkinson's disease), and its variation is also a symptom in deterioration of mental health: depression, anxiety, or suicide (Bedi et al., 2015; Cummins et al., 2015; Laske et al., 2015; Lopez-de-Ipina et al., 2013). Thus, variations of the speech signal are often used to study mental health through classic characteristics, such as prosody, pitch, acoustic formants, and energy. In other words, biomarkers/predictors of mental health status can be obtained from a voice signal.

Speech analysis is widely also used to study and precisely measure mental illness, including depression, suicide, and anxiety (Alghowinem et al., 2013; Alonso et al., 2015; Bedi et al., 2015; Cummins et al., 2013, 2015; France et al., 2000; Helfer et al., 2013; Hönig et al., 2014; Laske et al., 2015; Lopez-de-Ipina \& Barroso, 2017; 
Lopez-de-Ipina et al., 2013; Moore et al., 2004; Mundt et al., 2007; Ozdas et al., 2004; Quatieri \& Malyska, 2012; Williamson et al., 2014).

Regarding automatic analysis of spontaneous speech, some of the most typical features have been used to assess psychopathology and positive mental health: pitch, basic frequency F0, energy, frequency components, cepstrums, Mel-cepstrums (MFCC), intensity, harmonics, noise, shimmer, jitter, and their variants. Such features accurately measure the vocal generation system (muscles, oral tract, oral chords, joints, wind flow), as well as brain function. The features were extracted from the speech signal using MATLAB Toolbox (Mathworks, 2020) and PRAAT Program (Boersma \& Weenink, 2020).

\section{Data Processing}

First, descriptive statistics were obtained for the sample, and the reliability of the scales was then analyzed using Cronbach's alpha. Pearson correlational analysis was performed to examine the relationship between the measured variables. In order to compare older parents of Basque prisoner and those from the general Basque population, an independent samples $t$-test was carried out on the variables under analysis. The effect size test was performed, considering Cohen's $d$, and the following parameters were used in the statistically significant results $(p<0.05)$ : small effect size (value 0.20 ), medium (value 0.50), and large (0.80) (Cohen, 1992). The chi-square test was used to compare the distribution of the two groups according to their level of depression (minimal, mild, moderate, and severe). Effect size was measured using the Phi statistic, where the following parameters were used: small effect size (value 0.10), medium (value 0.30), and large (0.50) (Cohen, 1992). These analyses were run using SPSS 24.0 software.

\section{Results}

Internal consistency of the scales used in the study was good for all variables. Specifically, Cronbach's alpha was 0.83 for the BDI-II; 0.76 for the GASD; 0.79 for the PSS-14; and 0.89 for the MHC-SF. The positive mental health subscales also showed good-acceptable reliability ( 0.79 for emotional well-being; 0.69 for psychological well-being; and, finally, 0.86 for social well-being).

With respect to the relationships between the measured variables, they were as theoretically expected (see Table 1). Specifically, positive correlations were found between depression, anxiety, and stress, and negative correlations between the aforementioned variables and overall positive mental health score.

All correlations were statistically significant $(p<0.01)$.

In relation to the main objective of the study, prisoners' parents' scores in all variables were compared with those of the control group and significant differences were found in depression, anxiety, stress, and positive mental health (see Table 2). 
Table 1 Correlations between psychopathology (depression, anxiety, and stress) and positive mental health measures (Pearson's coefficient)
Table 2 Averages and standard deviations in psychopathology indicators (prisoners' parents versus control group)

\begin{tabular}{llll}
\hline & Anxiety & Stress & $\begin{array}{l}\text { Positive } \\
\text { mental } \\
\text { health }\end{array}$ \\
\hline Depression & 0.59 & 0.75 & -0.37 \\
Anxiety & & 0.70 & -0.29 \\
Stress & & & -0.41 \\
\hline
\end{tabular}

\begin{tabular}{llrl}
\hline $\begin{array}{l}\text { Psychopathology } \\
\text { indicators }\end{array}$ & Group & $M$ & $S D$ \\
\hline Depression & Prisoners' parents & 14.27 & 8.42 \\
$(0-63)$ & Control & 8.53 & 4.92 \\
Anxiety & Prisoners' parents & 2.02 & 1.54 \\
$(0-4)$ & Control & 0.91 & 1.10 \\
Stress & Prisoners' parents & 24.09 & 9.22 \\
$(0-56)$ & Control & 18.24 & 7.32 \\
\hline
\end{tabular}

In relation to the first hypothesis, as expected, prisoners' parents reported a higher level of depression than the control group parents, with a large effect size [ $t$ $(70.95)=3.94 ; p<0.001 ; d=0.84]$. Both groups of parents were distributed differently across the four categories of the BDI-II (minimal, mild, moderate, and severe depression) $\left(\chi^{2}(3)=16.30 ; p=0.001\right.$; Phi $=0.43$ ) (see Table 3). In particular, $51.1 \%$ of the prisoners' parents were in the minimum depression category; $24.4 \%$ in mild depression; $17.8 \%$ in moderate depression; and $6.7 \%$ in severe depression. With respect to the control group, $88.9 \%$ of the subjects were in the minimum depression category; $8.9 \%$ in mild depression; and $2.2 \%$ in moderate depression. No participant in this group presented severe depression. The control group showed an average score corresponding to the minimum depression $(M=8.53)$, while prisoners' parents showed an average score of mild depression $(M=14.27)$.

Similar results were found in relation to anxiety. Prisoners' parents reported a higher level of anxiety $(M=2.02)$ than parents in the control group $(M=0.91)$, with a large effect size $[t(79.65)=3.93 ; p<0.001 ; d=0.84]$. The subjects in the control group did not reach the minimum level of anxiety, while prisoners' parents did.

In terms of stress, the prisoners' parents reported a higher level of stress $(M=24.09)$ their counterparts in the control group $(M=18.24)$, with a mediumlarge effect size $[t(88)=3.33 ; p=0.001 ; d=0.71]$.

In relation to the second hypothesis, as expected, significant differences were found in positive mental health (see Table 4). In particular, prisoners' parents reported a lower level of mental health $(M=45.69)$ than those in the control group $(M=50.29)$, with a medium effect size $[t(88)=-2.37 ; p=0.020 ; d=0.51]$. Analyzing the subscales of positive mental health, significant differences were found in emotional well-being, with a large effect size $[t(83.87)=-4.08 ; p<0.001$; $d=0.88]$. In social well-being, trend differences were found $[t(88)=-1.74$; 
Table 3 Depression level by group (prisoners' parents versus control group)

\begin{tabular}{llll}
\hline & $\begin{array}{l}\text { Prisoners' } \\
\text { parents }\end{array}$ & Control group & Total \\
\hline Depression level & $\%$ & $\%$ & $\%$ \\
Minimal & 51.1 & 88.9 & 70.0 \\
Mild & 24.4 & 8.9 & 16.7 \\
Moderate & 17.8 & 2.2 & 10.0 \\
Severe & 6.7 & & 3.3 \\
Total & 100 & 100 & 100 \\
\hline
\end{tabular}

$p=0.086 ; d=0.41]$, while no difference was found in psychological well-being $[t$ $(88)=-1.25 ; p=0.215 ; d=0.27]$.

With the aim of analyzing and confirming the results for our objective, we carried out automatic analysis of the spontaneous speech. Table 5 shows significant differences in voice analysis features related to anxiety and depression in the prisoners' parents' scores when compared with the control group. As mentioned, after completing the questionnaire, participants were asked to talk about a positive event they had recently experienced. In this context, the prisoners' parents considered the improvement in the prisoners' situation as a generally positive event in their life and described it with great pain as a dramatic situation. This sign of adversity can usually be concealed but not when directly describing the situation in spontaneous speech conditions. As a result, in the speech analysis, the following alterations appear in the selected features: the voice became lower, frequency variations started at low frequencies and the voice broke and they spoke with less energy, even expressing symptoms of fatigue. They wanted to speak about these life experiences, but because it was so painful for them, sadness and fatigue had a clear impact on the flow of the voice. This difference was statistically significant as is shown in the following results of the Student's $t$-test on voice features: F0_std $[t(79)=3.16$; $p<0.002 ; d=13.96]$; MFCC5_mean $[(t(79)=3.24 ; p<0.002 ; d=0.73]$; MFCC7_ mean $[(t(79)=3.53 ; p=0.001 ; d=0.61]$, with a medium-large effect size.

Table 4 Group averages and standard deviations in positive mental health (prisoners' parents versus control group)

\begin{tabular}{llrl}
\hline & Group & $M$ & $S D$ \\
\hline Positive mental health (14-70) & Prisoners' parents & 45.69 & 9.31 \\
& Control & 50.29 & 9.07 \\
Emotional well-being (1-5) & Prisoners' parents & 3.07 & 0.88 \\
& Control & 3.76 & 0.70 \\
Psychological well-being (1-5) & Prisoners' parents & 3.53 & 0.81 \\
& Control & 3.74 & 0.75 \\
Social well-being (1-5) & Prisoners' parents & 3.05 & 0.64 \\
& Control & 3.31 & 0.75 \\
\hline
\end{tabular}


Table 5 Some features of the automatic speech analysis

\begin{tabular}{llllc}
\hline Feature & Group & $N$ & $M$ & $S D$ \\
\hline F0_std (in Hz) & Prisoners' parents & 39 & 54.09 & 20.17 \\
& Control group & 42 & 67.79 & 18.38 \\
Energy & Prisoners' parents & 39 & 31.02 & 28.56 \\
& Control group & 42 & 80.19 & 193.94 \\
Number of voicing breaks & Prisoners' parents & 39 & 41.38 & 32.95 \\
& Control group & 42 & 49.30 & 37.1 \\
MFCC5_mean & Prisoners' parents & 39 & 0.090 & 0.95 \\
& Control group & 42 & 0.642 & 1.06 \\
MFCC7_mean & Prisoners' parents & 39 & 0.35 & 0.6 \\
& Control group & 42 & 0.26 & 1.4 \\
\hline
\end{tabular}

\section{Discussion}

This study investigated secondary prisonization outcomes in over 65-year-old parents of prisoners incarcerated far from their homes. Our results supported the hypotheses of the study. In relation to the first hypothesis, as compared with the control group, parents with sons/daughters in remote prisons showed higher depressive, anxious, and stress-related symptomatology. In relation to the second hypothesis on positive mental health, parents of individuals incarcerated in remote prisons showed lower levels of positive mental health than parents with no incarcerated adult children.

Regarding the contributions of the present study, first, the study shows the relationship between secondary prisonization and mental health in an integrated way. Mental health is frequently explained exclusively in terms of psychopathological symptomatology. This research went beyond this reductive approach and integrated both psychopathological and salutogenic positive mental health perspectives currently addressed in mental health research. Second, the present study assessed the objective combining two different methodologies, that is, a descriptive methodology and an automatic analysis of spontaneous speech. This is a strength of the present research, as automatic analysis of spontaneous speech complements and contributes to descriptive analysis. In fact, when using selfreport questionnaires, participants are unable to express themselves freely and must follow the items established in the questionnaires. In contrast, speech analysis allows participants to explain whatever they want, with this being an objective indicator and permitting researchers to detect features that cannot be detected with self-questionnaires.

Finally, this study addressed a research topic that has largely been ignored: the effects of secondary prisonization on the mental health of older parents whose sons or daughters are incarcerated in remote prisons. Thus, the study makes a significant contribution to the fields of mental health and secondary prisonization.

As for the limitations of the study, the small sample size should be mentioned. In fact, many parents aged over 65 years had health problems and were unable to 
participate in the project. In other cases, some parents decided not to participate in the study for other reasons (i.e., they were not available and they thought it was unnecessary). Thus, and taking into account the small sample size, the results should be interpreted with caution.

Related to future lines of research, it would be interesting to assess gender differences between parents of imprisoned daughters/sons in order to better understand how secondary prisonization affects mental health. Likewise, other variables, such as self-esteem or resilience, could be measured in this sample with the aim of improving the parents' situation. Finally, and in light of the above, intervention programs could be designed to help parents in this specific population.

In sum, this study showed that older parents of Basque prisoners, due to the secondary prisonization they experience, have lower levels of mental health than ordinary parents of their age. Many ways to improve the mental health of older parents with incarcerated adult children could be implemented: for example, promoting a more attentive psychological or psychiatric assistance for this group. Indeed, specific groups of people with mental health problems usually demand (public) personalized assistance. Nevertheless, older parents of Basque inmates in remote prisons might constitute a different case because, strictly speaking, they do not suffer an "illness," but a situation that brings suffering to their lives in terms of stress, depression, and anxiety. Therefore, a more direct and easier way to manage the problem would be to implement the necessary changes in penitentiary policies with the purpose of discontinuing remote imprisonment. Changing the stressful situation experienced by these older parents would help improve their mental health.

Acknowledgements The authors would like to thank all the people that contribute to carry out the present research, Concretely, the elderly parents that participated in the study; the interviewers that helped carrying out the field work and the ETXERAT association, who helped authors getting the sample. Many thanks!!!

Funding Open Access funding provided thanks to the CRUE-CSIC agreement with Springer Nature.

Data Availability Data sharing is not applicable for ethical reasons included in the approval of the Ethical Committee for Research Involving Human Beings of the researchers' University.

\section{Declarations}

Conflict of Interest The authors declare that they have no conflict of interest.

Ethics Approval Approval of Ethical Committee for Research Involving Human Beings of the researchers' University was obtained (M10/2018/167).

Informed Consent.

Informed consent was obtained from all individual participants included in the study.

Open Access This article is licensed under a Creative Commons Attribution 4.0 International License, which permits use, sharing, adaptation, distribution and reproduction in any medium or format, as long as you give appropriate credit to the original author(s) and the source, provide a link to the Creative Commons licence, and indicate if changes were made. The images or other third party material in this article are included in the article's Creative Commons licence, unless indicated otherwise in a credit line to the material. If material is not included in the article's Creative Commons licence and your intended use is 
not permitted by statutory regulation or exceeds the permitted use, you will need to obtain permission directly from the copyright holder. To view a copy of this licence, visit http://creativecommons.org/licen ses/by/4.0/.

\section{References}

Aiello, B. L., \& McCorkel, J. A. (2018). 'It will crush you like a bug': Maternal incarceration, secondary prisonization, and children's visitation. Punishment \& Society, 20(3), 351-374. https://doi.org/10. $1177 / 1462474517697295$

Alghowinem, S., Goecke, R., Wagner, M., \& Epps, J. (2013). A comparative of different classifiers for detecting depression from spontaneous speech. In: Proceedings of ICASSP. Vancouver, Canada, pp. 8022-8026.

Alonso, J. B., Cabrera, J., Medina, M., \& Travieso, C. M. (2015). New approach in quantification of emotional intensity from the speech signal. Expert Systems with Applications, 42(24), 9554-9564. https://doi.org/10.1016/j.eswa.2015.07.062

American Psychiatric Association. (2013). DSM-5: Diagnostic and statistical manual of mental disorders (5th ed.). American Psychiatric Publishing.

Beck, A. T., Steer, R. A., \& Brown, G. K. (1996). Manual for the Beck Depression Inventory-II. Psychological Corporation.

Bedi, G., Carrillo, F., Cecchi, G. A., Fernández-Slezak, D., Sjgman, M., Mota, N. B., et al. (2015). Automated analysis of free speech predicts psychosis onset in high-risk youths. Schizophrenia, 1, 1-7.

Boersma, P. \& Weenink, D. (2020). Praat: Doing phonetic by computer. [Computer program]. Version 6.1.20beta, Amsterdam: University of Amsterdam. http://www.praat.org/ Accessed June 2020.

Cohen, J. (1992). A power primer. Psychological Bulletin, 112(1), 155-159.

Cohen, S., Kamarck, T., \& Mermelstein, R. (1983). A global measure of perceived stress. Journal of Health and Social Behaviour, 24, 385-396.

Comfort, M. L. (2003). In the tube at San Quentin: The "Secondary prisonization" of women visiting inmates. Journal of Contemporary Ethnography, 32(1), 77-107. https://doi.org/10.1177/08912 41602238939

Cummins, N., Scherer, S., Krajewski, J., Schnieder, S., Epps, J., \& Quatieri, T. F. (2015). A review of depression and suicide risk assessment using speech analysis. Speech Communication, 71, 10-49. https://doi.org/10.1016/j.specom.2015.03.004

Cummins, N., Epps, J., Sethu, V., Breakspear, M., \& Goecke, R. (2013). Modeling spectral variability for the classification of depressed speech. Proceedings of Interspeech. ISCA, Lyon, France, pp. 857-861.

Duch, F. R., Ruiz, L., Gimeno, D., \& Allué, B. (1999). Psicometría de la ansiedad, la depresión y el alcoholismo en atención primaria. Revista Española De Medicina De Familia, 3, 209-225.

Etxerat, (2018). Urrunketa: Kalte eta eraginak adin nagusiko pertsonengan. Bilbao: Etxerat.

European Court of Human Rights (2016). Judgments and decisions of 14 January 2016. Retrieved from https://hudoc.echr.coe.int.

France, D. J., Shiavi, R. G., Silverman, S., Silverman, M., \& Wilkes, M. (2000). Acoustical properties of speech as indicators of depression and suicidal risk. IEEE Transactions on Biomedical Engineering, 47, 829-837.

García-Campayo, J., Zamorano, E., Ruiz, M. A., Pardo, A., Pérez-Páramo, M., López-Gómez, V., Freire, O., \& Rejas, J. (2010). Cultural adaptation into Spanish of the generalized anxiety disorder-7 (GAD7) scale as a screening tool. Health and Quality of Life Outcomes, 8, 8.

Goldberg, D., Bridges, K., Duncan-Jones, P., \& Grayson, D. (1988). Detecting anxiety and depression in general medical settings. BMJ (clinical Research Ed.), 297(6653), 897-899.

Green, K. M., Ensminger, M. E., Robertson, J. A., \& Juon, H. (2006). Impact of adult sons' incarceration on African American mothers' psychological distress. Journal of Marriage and Family, 68(2), 430-441. https://doi.org/10.1111/j.1741-3737.2006.00262.x

Helfer, B. S., Quatieri, T. F., Williamson, J. R., Mehta, D. D., Horwitz, R., \& Yu, B. (2013). Classification of depression state based on articulatory precision. Proceedings of Interspeech, 2172-2176. 
Hönig, F., Batliner, A., Nöth, E., Schnieder, S., \& Krajewski, J. (2014). Automatic modelling of depressed speech: Relevant features and relevance of gender. Proceedings of Interspeech. Singapore, pp.1248-1252.

Intxaurbe, J. R., Ruiz, E. J., \& Urrutia, G. (2017). Urruntzeko espetxe-politikak presoen familengan duen eraginari buruzko txostena. Bilbao: Instituto de Derechos Humanos Pedro Arrupe.

Keyes, C. L. M. (2002). The mental health continuum: From languishing to flourishing in life. Journal of Health and Social Behavior, 43(2), 207-222. https://doi.org/10.2307/3090197

Keyes, C. L. M. (2005). Mental illness and/or mental health? Investigating axioms of the complete state model of health. Journal of Consulting and Clinical Psychology, 73(3), 539-548. https://doi.org/10. 1037/0022-006X.73.3.539

Laske, C., Sohrabi, H. R., Frost, S. M., Lopez-de-Ipina, K., Garrard, P., Buscema, M., et al. (2015). Innovative diagnostic tools for early detection of Alzheimer's disease. Alzheimer's \& Dementia, 11(5), 561-578. https://doi.org/10.1016/j.jalz.2014.06.004

Lopez-de-Ipina, K., \& Barroso, N. (2017). Bioinspired intelligence for machine learning. Neurocomputing, 255, 1-2.

Lopez-de-Ipina, K., Alonso, J. B., Travieso, C., Solé-Casals, J., \& Egiraun, H. (2013). On the selection of non-invasive methods based on speech analysis oriented to automatic Alzheimer disease diagnosis. Sensors, 13(5), 6730-6745.

Mackinnon, A., Christensen, H., Jorm, A. F., Henderson, A. F., Scott, R., \& Korten, A. E. (1994). A latent trait analysis of an inventory designed to detect symptoms of anxiety and depression using an elderly community sample. Psychological Medicine, 24(4), 977-986.

Mathworks (2020). MATHLAB toolbox. https://www.mathworks.com/products/matlab.html. Accessed June 2020.

Moore, E., Clements, M., Peifer, J., \& Weisser, L. (2004). Comparing objective feature statistics of speech for classifying clinical depression. 26th Annual International Conference of the IEEE Engineering in Medicine and Biology Society, 2004, IEMBS '04, pp. 17-20.

Mundt, J. C., Snyder, P. J., Cannizzaro, M. S., Chappie, K., \& Geralts, D. S. (2007). Voice acoustic measures of depression severity and treatment response collected via interactive voice response (IVR) technology. Journal of Neurolinguistics, 20, 50-64.

Murray, J., Farrington, D., \& Sekol, I. (2012). Children's antisocial behaviour, mental health, drug use and educational performance after parental incarceration. Psychological Bulletin, 138, 175-210. https://doi.org/10.1037/a0026407

Observatori del Sistema Penal i els Drets Humans (2006). La cárcel en el entorno familiar. estudio de las repercusiones del encarcelamiento sobre las familias: Problemáticas y necesidades. Barcelona: Quaderns de Barcelona.

Olivos, P., Galán, A., \& Santos, A. (2014). Relations between workload, work attitudes and psychological variables in Spanish university professors. Procedia-Social and Behavioural Sciences Journal, 140(22), 128-132. https://doi.org/10.1016/j.sbspro.2014.04.397

Ozdas, A., Shiavi, R. G., Silverman, S. E., Silverman, M. K., \& Wilkes, D. M. (2004). Investigation of vocal jitter and glottal flow spectrum as possible cues for depression and near-term suicidal risk. IEEE Transactions on Biomedical Engineering, 51, 1530-1540.

Pedrero-Pérez, E. J., Ruiz-Sánchez, J. M., Lozoya-Delgado, P., Rojo-Mota, G., Llanero-Luque, M., \& Puerta-García, C. (2015). La "Escala de estrés percibido": Estudio psicométrico sin restricciones en población no clínica y adictos a sustancias en tratamiento. Behavioral Psychology / Psicología Conductual, 23(2), 305-324.

Peña, E. K., Lima, S. E., Bueno, G. A., Aguilar, M. E., Keyes, C. L. M., \& Arias, W. P. (2017). Reliability and validity of the mental health continuum (MHC-SF) in the Ecuadorian contexts. Ciencias Psicológicas, 11(2), 223-232.

Quatieri, T. F. \& Malyska, N. (2012). Vocal-source biomarkers for depression: A link to psychomotor activity. Proceedings of Interspeech. ICSA, Portland, USA, pp. 1059-1062.

Remor, E. (2006). Psychometric properties of a European Spanish version of the perceived stress scale (PSS). The Spanish Journal of Psychology, 9(1), 86-93.

Sanz, J., \& Vázquez, C. (2011). BDI-II: Inventario de Depresión de Beck-II. Pearson Educación.

Sanz, J., Navarro, M. E., \& Vázquez, C. (2003). Adaptación española del Inventario para la Depresión de Beck II (BDI-II): 1. Propiedades psicométricas en estudiantes universitarios. Análisis y Modificación de Conducta, 29(124), 239-288. 
Sanz, J., Perdigón, L. A., \& Vázquez, C. (2003). Adaptación española del Inventario para la Depresión de Beck II (BDI-II): 2. Propiedades psicométricas en población general. Clínica y Salud, 14(3), 249-280.

Sanz, J., García-Vera, M. P., Espinosa, R., Fortún, M., \& Vázquez, C. (2005). Adaptación española del Inventario para la Depresión de Beck II (BDI-II): 3. Propiedades psicométricas en pacientes con trastornos psicológicos. Clínica y Salud, 16(2), 121-142.

Spain. Organic Law of the 5th of October 1979, General Penitentiary Law. Boletín Oficial del Estado, 5 de octubre de 1979, number 239, pp. 23180-23186.

Taylor, S. E. (1990). Health psychology: The science and the field. The American Psychologist, 45(1), 40-50.

Williamson, J., Quatieri, T., Helfer, B., Ciccarelli, G., \& Mehta, D.D. (2014). Vocal and facial biomarkers of depression based on motor incoordination and timing. Proceedings of the 4th ACM International Workshopon Audio/Visual Emotion Challenge (AVEC '14). ACM, Orlando,Florida, USA, pp. 65-72.

World Health Organization (2001). Strengthening mental health promotion. Geneva, World Health Organization (Fact sheet, No. 220).

World Health Organization (2018). ICD-11. International classification of diseases for mortality and morbidity statistics (11th Revision). Geneva, World Health Organization. https://icd.who.int/brows e11/1-m/en. Accessed June 2020.

World Prison Brief (2021). World Prison Brief Data. Retrieved from https://www.prisonstudies.org/. 